\title{
Opinión de padres y entrenadores sobre el reglamento en la competición de fútbol en Categoría alevín
}

Parents and coaches' opinions about the rules in football competition in the under 12 category

\author{
Juan Miguel Vicente-Aroca \\ Universidad de Murcia, Facultad de Ciencias del deporte \\ Jesús Salado-Tarodo (1) \\ Fundación San Pablo Andalucía CEU \\ jsalado@ceuandalucia.es
}

\begin{abstract}
Resumen
El objetivo del presente estudio fue conocer la opinión de los padres y entrenadores sobre el reglamento y el sistema de competición actual en fútbol en categoría alevín. Se desarrolló un diseño de tipo cualitativo, en el que se realizaron entrevistas semiestructuradas en las que se registraba la opinión de padres y entrenadores, en primer lugar de un campeonato con las reglas oficiales (Campeonato 1) y un siguiente torneo con reglas modificadas (Campeonato 2). La muestra fue de 33 padres y 4 entrenadores de fútbol. Se obtuvieron los siguientes resultados del análisis de las entrevistas: a) tan solo el $57 \%$ de los padres están satisfechos con la eficacia que producen las reglas actuales en el juego; b) el grupo de entrenadores no se halla nada satisfecho con el número de jugadores (8vs8) y con la eficacia de dichas reglas en el juego (25\%); c) el $90 \%$ de los padres entrevistados en el segundo campeonato están de acuerdo en que la modificación de las reglas produce un aumento de la participación de los jugadores; d) los padres consideran que las modificaciones del número jugadores y reducción del espacio no se adaptan a esta categoría, y no las utilizarían; y e) el 75\% de los entrenadores prefieren las nuevas reglas modificadas en cuanto a dimensiones del campo y a la reducción del número de jugadores a 5 vs5.
\end{abstract}

Palabras claves: fútbol, reglas, padres, entrenadores, satisfacción.

\begin{abstract}
The objective of this study was to know the parents and coaches opinion about the rules and the current competition system in football in under 12 category. A qualitative design was developed, in which semi-structured interviews were carried out in which the opinion of parents and coaches was recorded, first of a tournament with the official rules (Tournament 1) and a subsequent tournament with modified rules (Tournament 2). The sample consisted of 33 parents and 4 football coaches. The following results were obtained from the analysis of the interviews: a) only $57 \%$ of the parents are satisfied with the effectiveness of the current rules in the game; b) the group of coaches is not at all satisfied with the number of players (8vs8) and with the effectiveness of these rules in the game (25\%); c) $90 \%$ of the parents interviewed in the second championship agree that the modification of the rules leads to an increase in player participation; d) the parents consider that the changes in the number of players and reduction of space are not adapted to this category, and would not use them; and e) $75 \%$ of the coaches prefer the new modified rules in terms of field dimensions and reduction of the number of players to $5 \mathrm{vs} 5$.
\end{abstract}

Keywords:football, rules, parents, coaches, satisfaction.

Recibido: 8-oct-2019

Aceptado: 12-nov-2019

Publicado: 20-nov-2019

Como citar (apa): Vicente-Aroca J.M. y Salado-Tarodo J. (2020). Opinión de padres y entrenadores sobre el reglamento en la competición de fútbol en categoría alevín. JUMP, (1), 17-25.

Como citar (vancouver): Vicente-Aroca J.M. y Salado-Tarodo J. Opinión de padres y entrenadores sobre el reglamento en la competición de fútbol en categoría alevín. JUMP. 2020; (1), 17-25. 


\section{Introducción}

La adaptación de los deportes a las necesidades de los niños en su iniciación ha sido objeto de estudio en los últimos años. Tanto es así, que se puede observar una evolución de una metodología de la enseñanza más acorde hacia las necesidades y características de los jugadores que lo practican, para facilitar la consecución de los objetivos del juego y aumentar la motivación (Gutiérrez y García-López, 2012; Mateo, Miranda y Cárdenas, 2016).

En fútbol base, así como en el resto de los deportes en etapas de iniciación, las adaptaciones reglamentarias fueron creadas con objetivos enfocados a la formación global del niño, al desarrollo de hábitos de vida saludable, de valores positivos y el desarrollo óptimo de una conducta creativa, así como al propiciar una primera toma de contacto real con la competición deportiva (Kjønniksen, Anderssen, y Wold, 2009).

No obstante, la relación de los elementos estructurales que conforman algunos deportes adaptados no está bien adaptada a las características de los niños y esto produce una mala formación integral del propio individuo (Mateo et al., 2016). Esto resulta de vital importancia, dado que influye negativamente en aspectos tan importantes como la autoeficacia del niño (Ortega, 2006a), el disfrute, la diversión, etc., teniendo en cuenta que existe una relación directa con la probabilidad de participar en deportes regularmente en el futuro (Dishman et al., 2005; MacDonald, Côté, Eys y Deakin, 2011; Ortega-Toro et al., 2018).

Ante esta situación, han ido desarrollándose nuevas corrientes metodológicas que incrementan la eficacia del proceso de enseñanza-aprendizaje en las primeras etapas deportivas (Keith, Duarte, Vanda y Luís, 2013; Ortega, 2006b). Un método muy recurrido ha sido el llamado "Small-Sided Games" (SSG o juegos reducidos). Actualmente, existe una extensa literatura científica que justifica la idoneidad de los SSG como medio para mejorar la condición física y el aprendizaje de elementos técnico-tácticos en fútbol (Hill-Hass, Coutts, Rowsell y Dawson, 2008; Sarmento et al., 2018).

En la línea de los SSG, dentro del deporte del fútbol, Casamichana y Castellano (2010) comprobaron que a medida que se aumentaban las proporciones del terreno de juego (en situaciones de 5 Vs 5), la distancia total recorrida, los intervalos realizados entre moderados y vigorosos, la velocidad máxima y frecuencia de sprint y la frecuencia cardíaca tomaban valores más altos, así como la percepción de esfuerzo. A su vez también apreciaron que disminuían las variables relacionadas con la producción de acciones técnico-tácticas como el número de controles, regates y lanzamientos a portería.

En esta misma línea, Abrantes et al. (2012) compararon dos formas jugadas ( $3 \vee 3$ y $4 \vee 4$ ) y comprobaron que la frecuencia cardíaca y la percepción subjetiva del esfuerzo fueron más altos en la modalidad 3v3. Sin embargo, la producción y eficacia de las acciones técnicas fueron similares en ambas situaciones de juego, resultados similares a los registrados en el trabajo de Sampaio et al. (2007), en el cual se compararon dos situaciones de juego reducidas (2v2 y 3v3).

Paralelamente, en un estudio de Joo, Hwang-Bo y Jee, (2016) se compararon distintas modalidades de juego en fútbol (8v8 y 11v11) en diferentes dimensiones del terreno de juego (68x47 y $75 \times 47$ ). La modalidad $8 \mathrm{v} 8$ tanto en espacio reducido como en el oficial, mostro valores altos en cuanto a pases y lanzamientos, así como en variables fisiológicas respecto a la modalidad $11 \mathrm{v} 11$, la cual produjo índices menores en los criterios analizados. En la misma tónica, en el trabajo realizado por Ørntoft et al. (2016) se analizaron la modalidad 7v7 y 8 v8 en jóvenes jugadoras de fútbol. En los resultados se observaron que tanto la producción de acciones técnicas, así como el éxito de las acciones durante el juego fue mayor en la modalidad más reducida. Sin embargo, no se encontraron diferencias estadísticamente significativas en las variables fisiológicas, aunque la distancia recorrida fue mayor en la modalidad 8v8.

Por otro lado, los SSG también han sido utilizados en combinación con la modificación de los elementos estructurales y reglas que lo configuran, como la superficie del terreno de juego. Así pues, Montoya et al. (2010) analizaron la carga fisiológica en la situación de 3v3 en fútbol, introduciendo tres espacios distintos $(15 \times 15$, $20 \times 20$ y $30 \times 30$ ) en los cuales, la mayor carga fisiológica obtenida se produjo en el terreno de mayores dimensiones. A su vez, Brito et al. (2012), analizaron criterios fisiológicos modificando el tipo de terreno (arena, césped artificial y asfalto) en situaciones reducidas de fútbol (5v5). Los índices de mayor distancia recorrida, así como la cantidad de Sprint se produjeron en asfalto. Sin embargo, la 
frecuencia cardíaca obtenida y la percepción del esfuerzo fueron mayores en césped y arena que en asfalto.

También existen investigaciones cuyo objeto de estudio se centra en analizar la organización del tiempo destinado a la tarea (Hill-Hass, Rowsell, Dawson y Coutts, 2009; Tessitore, Meeusen, Piacentini, Demarie y Capranica, 2006) o el tipo de interacción del entrenador con los jugadores (Rampinini et al., 2007).

En las etapas de desarrollo, el deporte debe adaptarse a los niños y la reglamentación se usa para adaptarlo a las capacidades físicas y cognitivas. En el fútbol, el uso de modificación de reglas no proporciona información suficiente para saber cuál es el impacto real que genera. La mayoría de los estudios realizados han analizado el impacto de estas modificaciones a las reglas en jugadores de campo sénior, con respecto a aspectos físicos y en situaciones de entrenamiento. Se conoce menos información sobre el impacto del desarrollo técnico y sobre el aprendizaje de los jugadores (Ortega et al., 2018). Además, se deben tener en cuenta las fuentes motivacionales durante el proceso de formación en la iniciación deportiva. En este sentido, cobran gran importancia dos pilares fundamentales que influyen en la personalidad y las necesidades individuales y situacionales que son consecuencia para la motivación hacia la práctica de actividad física como son los entrenadores y los padres (González et al., 2000).

Por un lado, con respecto al rol del entrenador en categorías de formación será definitivo por constituir la pieza principal en el proceso de enseñanza-aprendizaje. Tanto es así que SáenzLópez, et al. (2007) obtuvieron como resultados de su trabajo que los jugadores consideraban al entrenador como una pieza clave que realza la calidad de estos. Siguiendo esta misma línea, los niños consideran al entrenador como la principal fuente de diversión (Garn y Cothran, 2006; Supaporn y Griffin, 1998).

Por otro lado, en cuanto a los padres, son considerados como las principales figuras influyentes en la socialización primaria del niño (Marcén et al., 2011). En relación con el deporte competitivo, el entorno familiar es determinante tanto para la iniciación como para el tipo de relación del niño durante el período de formación respecto a la dedicación, persistencia, motivación y continuidad futura en la actividad física-deportiva. Según Marcén, et al. (2011) este efecto influyente aparece por la copia de modelos de referencia y la cantidad de apoyo recibido.

Por ello, destacar la importancia de conocer el punto de vista de dichos agentes inmersos en el proceso de formación y considerados como piezas fundamentales en el proceso formativo del jugador que permita esclarecer qué reglas deben ser modificadas (Cañadas, Giménez-Egido y Ortega, 2017; Cañadas y Ortega, 2017; Ortega et al., 2012).

El objetivo de este trabajo es conocer la opinión de entrenadores y padres tras practicar dos torneos de categoría alevín, uno con las reglas oficiales y otro con la modificación de algunos elementos estructurales (número de jugadores, porterías y dimensiones del campo).

\section{Método}

El diseño del presente estudio fue de tipo cualitativo, donde se realizaron entrevistas a padres y entrenadores de los jugadores participantes en dos torneos, con el objetivo de conocer el grado de satisfacción/insatisfacción tanto en un primer torneo donde las reglas eran las oficiales y actuales (Campeonato 1) como en un segundo torneo donde se modificaron las dimensiones del campo

Tabla 1. Características de los campeonatos 1 y 2 (Reglas oficiales y modificadas).

\begin{tabular}{|c|c|c|}
\hline Reglamentación & Campeonato 1 (Fútbol 8) & Campeonato 2 (Fútbol 5) \\
\hline Número de jugadores (Equipo) & 7 jugadores de campo + Portero & 5 jugadores de campo + Portero \\
\hline Dimensiones campo (m) & $58 \times 38$ & $38 \times 20$ \\
\hline Dimensiones porterías (m) & $6 \times 2$ & $3 \times 2$ \\
\hline Dimensiones área del portero (m) & $24 \times 9 \mathrm{~m}$ & $12 \times 6 \mathrm{~m}$ \\
\hline Ratio de m2 de campo por jugador & $314 \mathrm{~m} 2$ & $190 \mathrm{~m} 2$ \\
\hline Dimensiones del balón (n) & $N^{0} 4$ & $N^{0} 4$ \\
\hline Sustituciones & Ilimitadas & Ilimitadas \\
\hline Tiempo (minutos) & 2 Periodos de $20 \mathrm{~min}$ & 2 Periodos de $20 \mathrm{~min}$ \\
\hline
\end{tabular}

Fuente: elaboración propia. 
y porterías, el número de jugadores participantes y el sistema de competición (Campeonato 2), siendo ambos de categoría Alevín.

Las reglas realizadas en los diferentes campeoantos se aprecian en la tabla 1, y son las mismas que las utilizadas por Ortega-Toro et al. (2018). En concreto, en ambos torneos jugaron los mismos jugadores de cuatro equipos de categoría alevín, jugando todos contra todos. En el torneo 1 se jugaban siguiendo las normas oficiales de esa categoría, en el torneo 2, se jugaron los mismos partidos, pero dividiendo los equipos en dos subequipos de $5 \times 5$ jugando ambos equipos en un espacio menor.

La muestra objeto de estudio estuvo conformada por un total de treinta y tres padres, de los cuales ninguno había jugado al fútbol federado, y no tenía ninguna formación relativa al fútbol; y por cuatro entrenadores de los respectivos equipos participantes en el torneo.

Los instrumentos utilizados fueron dos entrevistas semiestructuradas. Las entrevistas utilizadas son las siguientes: a) Entrevista pretorneo: el objetivo de esta entrevista fue conocer la opinión de los padres y entrenadores sobre el reglamento de fútbol en categoría alevín; y b) Entrevista postorneo: el objetivo de esta entrevista fue conocer la opinión de los entrevistados tras experimentar una situación competitiva (torneo 2) en la que habían sido modificadas las dimensiones del campo y porterías, el número de jugadores participantes y el sistema de competición en fútbol base alevín.

Para el diseño de las entrevistas, en primer lugar, se partió de una revisión bibliográfica de la cual se creó la primera propuesta. Tras esta propuesta se generó un panel de expertos formado por seis profesores universitarios con una experiencia de 10 años tanto en la docencia universitaria como en el ámbito del entrenamiento en fútbol. Como resultado de los grupos de discusión, la entrevista pretorneo para los padres se concretó en 12 preguntas, mientras que la entrevista postorneo en 8 preguntas. Por otro lado, tanto la entrevista pretorneo como la entrevista postorneo a los entrenadores estaba formada por 8 preguntas. Todas las entrevistas estaban divididas en tres dimensiones: contenidos y aspectos relevantes del entrenamiento y el juego en esta etapa; el reglamento (cuestiones sobre reglas específicas de esta categoría) y la competición en estas edades.

El análisis de los datos procedentes de las entrevistas se ha desarrollado a partir del análisis del contenido, mediante el proceso de codificación. Los códigos fueron determinados por miembros del grupo de investigadores siguiendo un proceso mixto (deductivo-inductivo). La codificación fue llevada a cabo por dos codificadores. La fiabilidad de estos fue superior al $80 \%$, y la concordancia inter-codificadores, tomados dos a dos, a través del valor de kappa de Cohen fue de $k=.76$. Los datos procedentes de las entrevistas fueron tratados tanto desde un enfoque cualitativo como cuantitativo.

\section{Resultados}

En la tabla 2 se muestran los resultados referentes a las opiniones a favor que tienen los padres y entrenadores respecto a diferentes criterios del sistema reglamentario oficial de la categoría alevín.

Los resultados que reflejan la tabla 2 muestran que los padres están muy satisfechos con las dimensiones del campo, las reglas actuales, el número de jugadores y muestran una satisfacción general del $92 \%$. Por el contrario, están algo menos satisfechos con la seguridad en el fútbol alevín y con la eficacia de las reglas en el juego.

Tabla 2. Opinión de los padres y entrenadores a favor de las diferentes reglas del reglamento de fútbol actual en categoría alevín (Campeonato 1).

\begin{tabular}{|c|c|c|c|}
\hline & Padres & Entrenadores & Total \\
\hline Dimensión campo & $92,9 \%$ & $100 \%$ & $94,4 \%$ \\
\hline Dimensión porterías & $78,6 \%$ & $50 \%$ & $72,2 \%$ \\
\hline Número de jugadores & $85,7 \%$ & $25 \%$ & $72 \%$ \\
\hline Reglas & $100 \%$ & $50 \%$ & $88,8 \%$ \\
\hline Eficacia en el juego & $57,1 \%$ & $25 \%$ & $50 \%$ \\
\hline Satisfacción general & $92,9 \%$ & $50 \%$ & $83,3 \%$ \\
\hline Seguridad & $750 \%$ & ---- & $50 \%$ \\
\hline
\end{tabular}

Fuente: elaboración propia. 
En concreto casi el total de los padres están satisfechos con las dimensiones del campo y no verían bien la reducción, haciendo alusión a razones como "para el paso al fútbol 17 lo notarían demasiado. Ahora mismo es ideal" (Padre 10), excepto uno de ellos, el cual hace referencia a que es complicado meter gol desde zonas alejadas, y también con las dimensiones de las porterías, aunque en este caso han sido más padres los que no estaban conformes, aludiendo a razones como "Deberían aumentar el tamaño de las porterías" (Padre 3 y 9 ).

Igual ocurre en la variable número de jugadores, pues excepto dos padres que no les gusta la cantidad de participantes en el juego con las reglas oficiales debido a "Hacer más grupos, no tenerlos tan apiñados, que pudieran disfrutar de más tiempo de juego" (Padre 6), el resto están conformes dando razones como "Sería muy pequeño, tienen que tener espacio para hacer jugadas" (Padre 1) u "8 es idóneo, mejor para después dar el salto a 71 jugadores" (Padre 9).

No obstante, es de destacar que en la variable eficacia en el juego, disminuye considerablemente el porcentaje de padres satisfechos, dando propuestas al respecto relacionadas con elementos estructurales, como "aumentar el tamaño de las porterías" (Padre 3) o aludiendo al planteamiento táctico "en equipos donde hayan más medios" (Padre 9), "haciendo jugadas para llegar a portería y lanzar más cerca" (Padre 10), "haciendo potencia de tiro" (padre 11 y 13); al nivel de desarrollo del niño "es por el crecimiento, le cuesta por eso" (Padre 8).

Por otro lado, la tabla 2 señala que los entrenadores están totalmente satisfechos con las dimensiones del campo definidas por las reglas oficiales, proporcionando opiniones del tipo "...ahora con el fútbol 8 se ocupa mejor el espacio" (Entrenador 4), "En el fútbol 8 está bien, una ocupaciónmás equitativa delterreno..."(Entrenador 1), pero también se aprecian opiniones contrarias del tipo "cuando el campo es más grande hay más parones en el juego, los espacios no están todos ocupados, los jugadores están más distanciados, me gustan espacios más pequeños para que haya más interacción con el balón." (Entrenador 1). Por otro lado, no están nada satisfechos con la eficacia de las reglas en el juego y con el número de jugadores (8vs8), aludiendo a razones como "... debería haber más espacios entre líneas, debería haber 1 menos por cada equipo." (Entrenador 3)

Así, al analizar las opiniones de los entrenadores previas a la competición modificada, el aspecto con más predilección encontrado ha sido la dimensión del campo, justificando las respuestas en base a "Si es fútbol 8 el campo está bien" (Entrenador 2) y "Más o menos bien, ahora con el fútbol 8 se ocupa mejor el espacio" (Entrenador 4). En el lado contrario se siguen encontrando comentarios con respecto a la práctica del fútbol alevín en unas dimensiones menores aludiendo a razones como "...participan más en el juego, llegar a portería y lo que les motiva es colar gol y hay más oportunidades de colar gol." (Entrenador 3), "Hay mucho desnivel entre equipos, pero normalmente hay gente con poca fuerza y resistencia... perjudica ya que no llegan a tanta distancia." (Entrenador 4)

No obstante, existe una equidad en cuanto a la conformidad y disconformidad con las dimensiones de las porterías. Finalmente, aquellos que se oponen a las reglas aluden a razones de "No. Desde el tamaño de las porterías, el tiempo de juego, que se pudiese pedir algún tiempo muerto..." (Entrenador 4).

En la tabla 3 se aprecia la opinión favorable de los padres y entrenadores sobre las modificaciones realizadas en el Campeonato 2 .

Los resultados de la tabla 3 muestran que los padres están muy satisfechos con la

Tabla 3. Opinión de los padres y entrenadores a favor de las diferentes reglas modificadas de fútbol en categoría alevín (Campeonato 2)

\begin{tabular}{lccc}
\hline & Padres & Entrenadores & Total \\
\hline Dimensión campo & $27,3 \%$ & $75 \%$ & $40 \%$ \\
Dimensión porterías & $27,3 \%$ & $50 \%$ & $33,3 \%$ \\
Número de jugadores (5vs5) & $45,5 \%$ & $75 \%$ & $53,3 \%$ \\
Adaptación a las reglas & $27,3 \%$ & $50 \%$ & $33,3 \%$ \\
Eficacia en el juego & $36,4 \%$ & $50 \%$ & $40 \%$ \\
Satisfacción general & $100 \%$ & $50 \%$ & $86,6 \%$ \\
Participación & $90.9 \%$ & $75 \%$ & $86,6 \%$ \\
Motivación & $36,4 \%$ & $50 \%$ & $40 \%$ \\
\hline
\end{tabular}

Fuente: elaboración propia. 
participación de los jugadores al modificar las reglas (campeonato 2) y muestran una total satisfacción general con este campeonato. Sin embargo esto contrasta con la considerable disminución en cuanto a los valores del resto de variables referentes a las reglas modificadas, a la disminución del número de jugadores, a la dimensión de las porterías y del campo, incluso a la eficacia de los jugadores debido a "en 2 días no se pueden adaptar" (Padre 1), "hay menos goles" (Padre 2), "cuesta más meter goles" (Padre 4) o "Están acostumbrados a campo grande, portería grande" (Padre 5).

En cuanto a las razones encontradas respecto a las nuevas reglas implementadas que muestran su disconformidad, están las que aluden a "no me gusta que estén tan cerca, se pueden liar con las voces del otro campo" (Padre 5), "están más acostumbrados al tradicional y juegan con todos sus compañeros" (Padre 6), "veo que este campo es un poco pequeño" (Padre 7).

El alto porcentaje de insatisfacción en cuanto a la forma jugada de $5 v 5$ es debido a la alusión de los padres en relación con "lo veo demasiado pequeño, se va la pelota enseguida" (Padre 9), "lo veo para críos más pequeños" (Padre 11), "Pienso que en la edad que están entrando ya deben adaptarse a las características de fútbol 11 a la reglamentación de 11 que a la de fútbol 5". (Padre 2). Sin embargo, por otro lado, también se encuentran opiniones positivas a la forma de jugar del 5vs5, con comentarios del tipo "Está participando más en fútbol 5" (Padre 7)

Un aspecto positivo para destacar es que más del $90 \%$ de padres están de acuerdo en que con las modificaciones introducidas en el campeonato 2 , aumenta sustancialmente la participación de los jugadores que aluden a razones como "Sí. Lo veo más contento e inspirado hoy" (Padre 7), siendo uno el que no estaba en consonancia con el resto debido a "Sí pero negativamente, él está acostumbrado a espacios, a desbordar y correr y aquí está limitado, lo estoy viendo más espeso" (Padre 3).

Por otro lado, los datos de la tabla 3 señalan que los entrenadores muestran estar bastante satisfechos con las dimensiones del campo, número de jugadores y con la participación. Por el contrario, están algo menos satisfechos con la dimensión de porterías, adaptación a las reglas, eficacia de las nuevas reglas en el juego, la satisfacción en general con las nuevas reglas y la motivación que genera en los jugadores (50\%).
Así es de destacar que la gran mayoría prefieren, tras el campeonato modificado, la implementación de las nuevas dimensiones del campo y la reducción del número de jugadores a 5, justificando sus opiniones en que "Ha habido una incidencia mayor de los jugadores en el juego, probablemente se sientan que han participado más y con mayor satisfacción" (Entrenador 1), "Han tenido tiempo todos de jugar el mismo tiempo" (Entrenador 2), mientras que el entrenador 4 no quedó satisfecho puesto que "los críos están más acostumbrados a participar menos y no correr tanto ni parar arriba ni abajo. Hay menos satisfacción menos puntería y menos motivación".

Sin embargo, hay disparidad de opiniones en cuanto a la modificación de las dimensiones de la portería debido a "más difícil meter goles" (Entrenador 3) y una reticencia por parte de la mitad de los encuestados a la adaptación general del reglamento "Los críos van desorganizados, van detrás de la pelota" (Entrenador 4) o "es menos formativo porque no participan tanto en la elaboración de jugadas, es más directo" (Entrenador 3).

A pesar de los altos valores obtenidos en cuanto a las variables de dimensión de campo y del número de jugadores, se ha obtenido el 50\% en satisfacción general y en la eficacia que se produce en el juego, argumentando sus respuestas en relación a "En la participación, ya no hay una táctica, todos participan tanto arriba como abajo. La portería de fútbol 5 los porteros son demasiado grandes y en ocasiones demasiado pequeños para las de fútbol 8" (Entrenador 1).

\section{Discusión}

El objetivo del presente trabajo era analizar la opinión de entrenadores y padres tras la aplicación de un torneo de fútbol categoría alevín, en el que se modificó el reglamento y el sistema de competición.

Los padres, antes del torneo opinaron que estaban conformes con las reglas oficiales que contiene el actual reglamento en categoría alevín, manteniendo su idoneidad respecto a las características de los jugadores, señalando como principal aspecto a mejorar la eficacia de los jóvenes jugadores dentro de la dinámica de juego, especialmente en el tiro, aspecto que es considerado como importante en la mejora durante el proceso de iniciación deportiva (Abrantes et al., 
2012; Casamichana y Castellano, 2010; Joo et al., 2016; Sampaio et al., 2007).

En cambio, los entrenadores se presentan más críticos al respecto, principalmente al considerar la modalidad 8vs.8 como un número excesivo de jugadores participantes, siendo además las reglas el segundo aspecto con el que menos conforme se encuentran, aspecto altamente modificado en numerosos estudios para conseguir mejores resultados (Hill-Hass et al., 2008; Keith et al., 2013).

Tras la realización de la nueva competición, el cambio más notable observado por ambos colectivos que presentó mayor satisfacción fue el relacionado con el incremento de la participación. Aunque sólo en este estudio se evaluó la opinión de los sujetos mediante entrevistas, se obtuvieron iguales resultados que en estudios anteriores en relación con la modificación de la modalidad de juego (Abrantes et al., 2012; Casamichana y Castellano, 2010; Hill-Hass, 2008; Joo et al., 2016; Keith et al., 2013; Sampaio et al, 2007).

Siguiendo la misma línea, la reducción del número de jugadores ha sido asociada por los padres como una propuesta inadecuada, al contemplar un juego más desorganizado y con menor control táctico. Sin embargo, ha sido muy bien aceptada por parte de los entrenadores, al considerarla adecuada por la aparición de un mayor número de ocasiones de gol, de transiciones ataque-defensa, así como contactos con el balón, lo que según estudios previos conforman aspectos esenciales en la formación del joven jugador (Dishman et al., 2005; Gutiérrez y GarcíaLópez, 2012; Kjønniksen et al., 2009; MacDonald et al., 2011; Mateo et al., 2016).

El paso de la modalidad 8vs.8 a $5 \mathrm{vs.} 5$ ha sido la variable causante de alto grado de satisfacción de los entrevistados en relación a la participación y de aprendizaje, pues así se refleja en estudios anteriores relacionados con las situaciones de juego reducido (Abrantes et al., 2012; Casamichana y Castellano, 2010; Joo et al., 2016; Sampaio et al., 2007), al plantear un nivel de exigencia acorde a la edad y características de los jugadores (Calderon, Palao, Ortega, 2005; Gutiérrez y García-López, 2012; Mateo et al., 2016).

En cuanto a la modificación de las dimensiones del campo, las reacciones de los padres siguen manteniéndose negativas, aludiendo razones referentes a que el paso a la siguiente categoría sería más complicado o bien que tienen menos espacio para poder jugar. Dicha opinión contrasta con la obtenida en los entrenadores, los cuales fundamentan su idoneidad en la mayor incidencia de cada jugador sobre durante el desarrollo del juego, opiniones que concuerdan con las obtenidas en estudios que analizaron aspectos técnico-tácticos al modificar el espacio de juego (Brito et al., 2012; Joo et al., 2016; Montoya et al, 2010; Sampaio y Macas, 2012).

La reducción de las porterías no fue una modificación que produjo gran satisfacción en ambos colectivos, y, por ende, tampoco la eficacia. Los puntos negativos que consideraron en relación con las porterías fueron relacionados con el éxito en las acciones motrices, al argumentar que su reducción beneficiaba a los porteros y limitaba la eficacia en los lanzamientos. Sin embargo, el 50\% de los entrenadores aceptaron como buena dicha propuesta fundamentándose en que las proporciones de las porterías estaban más acordes y proporcionadas al desarrollo fisiológico de los jugadores, teniéndose en cuenta el hecho de que en estas categorías no se trataba de meter el máximo número de goles. En este sentido, el equipamiento deportivo adaptado puede proporcionar a los niños pequeños, que a menudo carecen de la fuerza necesaria para usar el equipamiento adulto de manera eficiente (restricción orgánica), la oportunidad de realizar las habilidades necesarias y, por lo tanto, encontrar la solución de movimiento óptimo cuando se juega en un partido, particularmente cuando las condiciones, como el clima, son menos favorables (restricción ambiental). Al hacerlo, esto puede facilitar el acoplamiento de los procesos de percepción-acción, que se considera esencial para los patrones de movimiento coordinados (Buszard, Reid, Masters y Farrow, 2016; Ortega-Toro et al., 2018).

Desde una perspectiva general, los padres se muestran más reticentes que los entrenadores en cuanto a la modificación de los elementos estructurales del juego en el fútbol de formación, en relación con los siguientes aspectos: cantidad de jugadores, proporción de las porterías y dimensiones del campo. Sin embargo, la satisfacción general del nuevo sistema de competición es altamente positiva.

Así pues, se hace necesario persistir en el planteamiento de este tipo de propuestas en el que se introduzcan cambios reglamentarios y que puedan ser observados y juzgados por un colectivo mayor, con el objetivo de concretar 
aquellos cambios más acordes para mejorar la formación de los niños y poder así ir introduciendo este tipo de modificaciones en el reglamento en el deporte base.

\section{Aplicaciones prácticas}

Los resultados del presente estudio, denotan en primer lugar, la necesidad que señalan tanto padres como entrenadores de que la competición debe estar adaptada a las necesidades y características de los jóvenes deportistas. Todos los agentes analizados estaban de acuerdo en este aspecto. Sin embargo, las dudas aparecen en señalar qué reglas son las que permiten realmente una adecuada adaptación. En este sentido, es necesario seguir realizando estudios que analicen la incidencia de modificaciones reglamentarias en la competición sobre las diferentes variables relacionadas con el proceso de formación del niños, tales como variables técnico-tácticas, físicas, psicológicas, sociológicas, etc. En cualquier caso, debe ser siempre la evidencia científica la que señale las propuestas a utilizar y los cambios a plantear, evidencias que, a nuestro parecer, deben proceder desde todos y cada uno de los diferentes agentes relacionados con los procesos de formación (entrenadores, jugadores, directivos, padres, psicólogos, preparadores físicos, etc.), si bien el peso de cada uno de ellos se deberá relativizar.

Únicamente a partir de estas evidencias, se podrá encontrar una competición deportiva realmente adaptada a las necesidades de los jóvenes jugadores. Serán los responsables políticos deportivos, los que deberán ser los precursores de trabajos de investigación de estetipo, para tomar decisiones consensuadas y sustentadas en evidencias científicas

\section{Referencias}

Abrantes, I., Nunes, I., Maçãs, M., Leite, M. \& Sampaio, J. (2012). Effects of the number of players and game type constrainsts on heart rate, rating of perceived exertion, and technical actions of small-sided soccer games. Journal of Strength and Conditioning Research, 26(4), 976-981. http://dx.doi.org/10.1519/JSC.0b013e31822dd398

Brito, J., Krustrup, P. \& Rebelo, A. (2012). The influence of the playing surface on the exercise intensity of small-sided recreational soccer games. Human Movement Science 31,946-956. http://dx.doi.org/10.1016/j.humov.2011.08.011

Buszard, T., Reid, M., Masters, R. \& Farrow, D. (2016). Scaling the equipment and play area in children's sport to improve motor skill acquisition: A systematic review. Sports medicine, 46(6), 829-843.

Calderón, A., Palao, J.M. \& Ortega, E. (2005). Incidencia de la forma de organización sobre la participación, el feedback impartido, la calidad de las ejecuciones y la motivación en la enseñanza de habilidades atléticas. Cultura, ciencia y deporte, 1(3), 145155 .

Cañadas, M. \& Ortega, E. (2017). Modificación de reglas y competición en baloncesto: opinión de padres y entrenadores. Revista de psicología del deporte, 26(1), 23-26.

Casamichana, D. \& Castellano, J. (2010). Time-motion, heart rate, perceptual and motor behaviour demands in small-sides soccer games: effects of pitch size. Journal of sports sciences, 28(14), 1615-1623. http://dx.doi.org/10.1080/02640414.201 0.521168

Dellal, A., Hill-Haas, S., Lago-Penas, C. \& Chamari, K. (2011). Smallsided games in soccer: amateur vs. professional players's physiological responses, physical, and technical activities. Journal of strength \& conditioning research, 25(9), 2371-2381. http://dx.doi.org/10.1519/JSC.0b013e3181fb4296

Dishman, R., Molt, R., Saunders, R., Felton, G., Ward, D., Dowda, M., Pate, R.R. (2005). Enjoyment mediates effects of a schoolbased physical-activity intervention. Medicine \& Science in Sports \& Exercise, 37(3), 478-487.

Garn, A. C. \& Cothran, D. J. (2006). The fun factor in physical education. Journal of Teaching in Physical Education, 25(3), 281-297.

González, G., Tabernero, B. \& Márquez, S. (2000). Análisis de los motivos para participar en fútbol y en tenis en la iniciación deportiva. Motricidad, 6, 47-66

Gutiérrez, D. \& García-López, L.M. (2012). Evaluación de la toma de decisiones por parte de alumnos de primaria en relación a contextos tácticos. New Approaches In Educational Research, 1, 2254-7339.

Hill-Haas, S. V., Coutts, A., Rowsell, G. \& Dawson, B. (2008). Variability of acute physiological responses and performance profiles of youth soccer players in small- sided games. Journal of science and medicine in sport, 11, 487-490. http://dx.doi.org/10.1016/j.jsams.2007.07.006

Hill-Haas, S. V., Rowsell, G. J., Dawson, B. T. \& Coutts, A. J. (2009). Acute physiological responses and time-motion characteristics of two small-sided training regimes in youth soccer players. Journal of strength \& conditioning research, 23(1), 111-115. http://dx.doi.org/10.1519/JSC.0b013e31818efc1a

Hugo Sarmento, Filipe Manuel Clemente, Liam David Harper, Israel Teoldo da Costa, Adam Owen \& António J. Figueiredo (2018): Small sided games in soccer - a systematic review International Journal of Performance Analysis in Sport, DOI: 10.1080/24748668.2018.1517288

Joo, C. H., Hwang-Bo, K. \& Jee, H. (2016). Technical and physical activities of small-sided games in young Korean soccer players. Journal of strength and conditioning research, 30(8), 21642173. http://dx.doi.org/10.1519/JSC.0000000000001319

Keith, D., Duarte A., Vanda C. \& Luís, V. (2013). How small-sided and conditioned games enhance acquisition of movement and decision-making skills. Exercise \& Sport Sciences Review, 41, 154-161. http://dx.doi.org/10.1097/JES.0b013e318292f3ec

Kjønniksen, L., Anderssen, N. \& Wold, B. (2009). Organized youth sport as a predictor of physical activity in adulthood. Scandinavian Journal of Medicine \& Science in Sports, 19(5), 646-654.

MacDonald, D., Côté, J., Eys, M. A. \& Deakin, J. (2011). The role of enjoyment and motivational climate in relation to the personal development of team sport athletes. The Sport Psychologist 25(1), 32-46.

Marcén, C., Gimeno, F., Gómez, C., Gutiérrez, H., Sáenz, A. \& Lacambra, D. (2011). La influencia de los padres en la práctica deportiva competitiva. (Material no publicado). Departamento 
de Psicología y Sociología: Universidad de Zaragoza.

Marrero, G., Martín-Albo, J. \& Núñez, J. L. (1997). Motivos, motivación y deporte. Las Palmas de Gran Canaria: Tesitex

Mateo, M. T., Miranda, M. T. \& Cárdenas, D. (2016). La línea de tres puntos y su influencia sobre la dinámica de juego en minibasket, el grado de satisfacción individual y competencia percibida de niños y niñas de la Región de Murcia. Sport Tk, 5 , 89-102.

Montoya, D., De Paz, J.A., Fernández, R., Mercé, J. \& Yagüe, J. M. (2010). Variabilidad de la carga fisiológica en los pequeños juegos de fútbol en función del espacio. Apunts: Educación física y deportes, 102, 70-77.

Ørntoft, C., Larsen, M. N., Andersen, T. B., Rasmussen, L. S., Póvoas, S. C., Randers, M. B. \& Krustrup, P. (2016). Technical actions, heart rate, and locomotor activity in $7 \mathrm{v} 7$ and $8 \mathrm{v} 8$ games for female youth soccer players. The Journal of Strength \& Conditioning Research, 30(12), 3298-3303. http://dx.doi.org/10.1519/JSC.0000000000001434

Ortega, E. (2006q). Autoeficacia y deporte. Sevilla: Wanceulen

Ortega, E. (2006b). La competición como medio formativo en el baloncesto. Sevilla: Wanceulen.

Ortega, E., Piñar, M. I., Salado, J., Palao, J. M. \& Gómez, M. A. (2012). Opinión de expertos y entrenadores sobre el reglamento de la competición infantil en baloncesto. Ricyde, 8(28), 142-150.

Ortega-Toro, E., Cañadas, M. \& Giménez-Egido, J.M. (2017). Incidencia de la modificación de reglas y sistema de competición sobre los niveles de satisfacción en jugadores de baloncesto under 14. Revista de psicología del deporte, 26(1), 59-64.

Ortega-Toro, E., García-Angulo, A., Giménez-Egido, J., GarcíaAngulo, F. and Palao, J. (2018). Effect of modifications in rules in competition on participation of male youth goalkeepers in soccer. International Journal of Sports Science \& Coaching, 13(6), 1040-1047.

Rampinini, E., Impellizzeri, F. M., Castagna, C., Abt, G., Chamari, K., Sassi, A. \& Marcora, S. M. (2007). Factors influencing physiological responses to small-sided soccer games. Journal of sports sciences, 25(6), 659-66. http://dx.doi. org/10.1080/02640410600811858

Sáenz-López, P., Jiménez, A. C., Giménez, F. J. \& Ibáñez, S. J. (2007). La auto percepción de la jugadoras de baloncesto expertas respecto a sus procesos de formación. Cultura Ciencia y Deporte, 7(3), 35-41.

Sampaio, J. \& Maçãs, V. (2012). Measuring tactical behaviour in football. International Journal Sports Medicine, 33(5), 395-401. http://dx.doi.org/10.1055/s-0031-1301320

Sampaio, J., Garcia, G., Macas, V., Ibanez, J., Abrantes, C. \& Caixinha, P. (2007). Heart rate and perceptual responses to $2 \times 2$ and $3 \times$ 3 small-sided youth soccer games. Journal of sports science and medicine, 6(Suppl 10), 121-122.

Santos, S.D., Memmert, D., Sampaio, J. (2006). The spawns of creative behavior in team sports: a creativity developmental framework. Frontier in Psychology, 7, 1282

Supaporn, S. \& Griffin, L. L. (1998). Undergraduate students report their meaning and experiences of having fun in physical education. Physical Educator, 55(2), 57-67.

Tessitore, A., Meeusen, R., Piacentini, M., Demarie, S. \& Capranica, L. (2006). Physiological and technical aspects of "6-a-side" soccer drills. Journal of sports medicine and physical fitness, $46,36-43$. 